\title{
Barnett relaxation in non-symmetric grains
}

\author{
Erald Kolasi ${ }^{1 \star}$ and Joseph C. Weingartner ${ }^{1}$ \\ ${ }^{1}$ Department of Physics and Astronomy, George Mason University, 4400 University Drive, Fairfax, VA 22030, USA
}

Accepted XXX. Received YYY; in original form ZZZ

\begin{abstract}
Barnett relaxation, first described by Purcell in 1979, appears to play a major role in the alignment of grains with the interstellar magnetic field. In 1999, Lazarian and Draine proposed that Barnett relaxation and its relative, nuclear relaxation, can induce grains to flip. If this thermal flipping is rapid, then the dynamical effect of torques that are fixed relative to the grain body can be greatly reduced. To date, detailed studies of Barnett relaxation have been confined to grains exhibiting dynamic symmetry. In 2003, Weingartner argued that internal relaxation cannot induce flips in any grains, whether they exhibit dynamic symmetry or not. In this work, we develop approximate expressions for the dissipation rate and diffusion coefficient for Barnett relaxation. We revisit the issue of internally induced thermal flipping, finding that it cannot occur for grains with dynamic symmetry but does occur for grains lacking dynamic symmetry.
\end{abstract}

Key words: dust, extinction - ISM: magnetic fields

\section{INTRODUCTION}

The discovery of starlight polarization nearly 70 years ago (Hall 1949; Hall \& Mikesell 1949; Hiltner 1949a,b) revealed that interstellar dust grains are nonspherical and aligned with the interstellar magnetic field. Yet we still do not have a complete, unambiguous theory of the alignment mechanism.

In seminal work, Purcell (1979) identified the process of "Barnett dissipation", in which rotational kinetic energy is converted into vibrational energy in paramagnetic grains. If a grain rotates steadily around a principal axis, then there is no Barnett dissipation. Otherwise, the grain's angular velocity $\omega$ is not constant as observed in the grain-body frame (i.e. the non-inertial frame in which the grain does not rotate). The microscopic spins in the paramagnetic material tend to align along the "Barnett-equivalent magnetic field" $\boldsymbol{B}_{\mathrm{BE}}=\omega / \gamma_{g}\left(\gamma_{g}\right.$ is the gyromagnetic ratio of the microscopic spins), but with a lag. As a result, energy is dissipated into heat, as in magnetic resonance.

Lazarian \& Roberge (1997) examined the inverse process, in which thermal fluctuations convert vibrational energy into rotational kinetic energy, deriving an expression for the diffusion coefficient that appears in the Fokker-Planck and Langevin equations describing Barnett relaxation (i.e. the combination of both dissipation and fluctuations).

Barnett relaxation is an internal process, unrelated to any external torques or other influences. Thus, the grain angular momentum $\boldsymbol{J}$ remains constant under Barnett relaxation. Lazarian \& Draine (1999a) introduced the concept of "thermal flipping", in which the sign of $\boldsymbol{J} \cdot \hat{\boldsymbol{a}}_{1}$ changes as a result of a Barnett fluctuation; $\hat{\boldsymbol{a}}_{1}$ is the principal axis of greatest moment of inertia. Thermal flipping can potentially have important implications for grain alignment, due to another major insight in Purcell (1979), namely the existence of systematic torques fixed relative to the grain body. These torques can spin the grain up to rapid rotation, making them impervious to disalignment via random collisions with gas particles. Each grain flip reverses the direction of the systematic torque in space (i.e. relative to an inertial frame). If flips occur in rapid succession, then the average systematic torque is zero and the grain may not be spun up after all. Lazarian \& Draine (1999a) refer to this condition as "thermal trapping". Noting the potential importance of nuclear paramagnetism in addition to electron paramagnetism, Lazarian \& Draine (1999b) examined "nuclear relaxation" and concluded that grains that are responsible for the observed starlight polarization (i.e. with sizes $<1 \mu \mathrm{m}$ ) are likely thermally trapped.

^ E-mail: ekolasi@masonlive.gmu.edu; jweinga1@gmu.edu

(C) 2015 The Authors 


\section{E. Kolasi \& J. C. Weingartner}

Weingartner (2009) revisited the treatment of Barnett relaxation in Lazarian \& Roberge (1997) and found a revised expression for the diffusion coefficient. With this revision, thermal flipping was not possible. Shortly thereafter, Hoang \& Lazarian (2009) found that flips resulting from collisions with gas particles likely occur sufficiently rapidly for grains to become trapped.

For simplicity, treatments of Barnett relaxation have focused on oblate grains with dynamic symmetry. That is, the moments of inertia associated with the principal axes $\hat{\boldsymbol{a}}_{1}, \hat{\boldsymbol{a}}_{2}$, and $\hat{\boldsymbol{a}}_{3}$ have $I_{2}=I_{3}$ and $I_{1}>I_{2}$. It seems unlikely that real interstellar grains would be characterized by dynamic symmetry. Thus, in this work, we examine Barnett relaxation for non-symmetric grains, with three different principal moments of inertia.

Although Weingartner (2009) computed the diffusion coefficient only for symmetric grains, he described general considerations that implied that thermal flipping would be impossible also for non-symmetric grains. However, we find that thermal flipping is possible for non-symmetric grains. In section 2, we briefly summarize and revisit the analysis in Weingartner (2009). We conclude that thermal flipping is not possible for symmetric grains, but not necessarily for the reason Weingartner (2009) identified. The argument applies only to symmetric grains and is mute regarding non-symmetric grains. In sections 3 and 4 , respectively, we develop approximate expressions for the Barnett dissipation rate and diffusion coefficient for non-symmetric grains. Section 5 describes the results of simulations of Barnett relaxation for a few shapes, exhibiting rapid thermal flipping. Section 6 summarizes the results.

\section{SYMMETRIC GRAINS}

For an oblate grain with dynamic symmetry, the solution of Euler's equation is simple. The component of the angular velocity along $\hat{\boldsymbol{a}}_{1}$, the principal axis of greatest moment of inertia, is constant with magnitude

$\omega_{\|}=\frac{J}{I_{1}} \cos \gamma$

where $\boldsymbol{J}$ is the angular momentum and $\gamma$ is the angle between $\boldsymbol{J}$ and $\hat{\boldsymbol{a}}_{1}$. There is also a component in the $\hat{\boldsymbol{a}}_{2}-\hat{\boldsymbol{a}}_{3}$ plane with magnitude

$\omega_{\perp}=\frac{J}{I_{2}} \sin \gamma$

that rotates with angular speed

$\omega_{\text {rot }}=\frac{J\left(I_{1}-I_{2}\right)}{I_{1} I_{2}} \cos \gamma$

Since the rotating component of the Barnett-equivalent field $B_{\mathrm{BE} \text {,rot }}=\omega_{\perp} / \gamma_{g}$, a direct analogy with the standard treatment of magnetic resonance offers an immediate expression for the rate at which the grain's rotational kinetic energy is dissipated:

$\left(\frac{d E}{d t}\right)_{\mathrm{Bar}}=-V \chi^{\prime \prime} B_{\mathrm{BE}, \mathrm{rot}}^{2} \omega_{\mathrm{rot}}$

where $V$ is the grain volume and $\chi^{\prime \prime}$ is the imaginary component of the magnetic susceptibility. When introducing this result, Purcell (1979) adopted the low-frequency susceptibility

$\chi^{\prime \prime} \approx \chi_{0} \omega_{\text {rot }} T_{2}$,

where $\chi_{0}$ is the static susceptibility and $T_{2}$ is the spin-spin relaxation time. This expression, as well as a more general result for arbitrary frequencies, can be inferred from the Bloch equations.

It is convenient to introduce a dimensionless measure of the rotational kinetic energy,

$q=\frac{2 I_{1} E}{J^{2}}$.

From equation (4), the corresponding dissipation rate is

$A(q)=\frac{d q}{d t}=-\tau_{\text {Bar }}^{-1}(q-1)\left(r_{2}-q\right)$

with

$\tau_{\mathrm{Bar}}=\frac{\gamma_{g}^{2} I_{1} I_{2}^{2}}{2 \chi_{0} V T_{2} J^{2}}$

and $r_{2}=I_{1} / I_{2}$. This expression for $A(q)$ is the drift coefficient in the Fokker-Planck and Langevin equations.

To find the diffusion coefficient $D(q)$, Lazarian \& Roberge (1997) and Weingartner (2009) demanded that the probability current

$S(q)=A(q) f(q)-\frac{1}{2} \frac{\mathrm{d}[f(q) D(q)]}{\mathrm{d} q}$ 
vanish for all $q$ when thermal equilibrium applies. Here $f(q)$ is the probability density. That is, $f(q)$ d $q$ is the probability that the dimensionless energy lies between $q$ and $q+\mathrm{d} q$. Thus,

$D(q)=\frac{1}{f_{\mathrm{TE}}(q)}\left[D(1) f_{\mathrm{TE}}(1)+2 \int_{1}^{q} A\left(q^{\prime}\right) f_{\mathrm{TE}}\left(q^{\prime}\right) \mathrm{d} q^{\prime}\right]$,

where $f_{\mathrm{TE}}(q)$ denotes the thermal-equilibrium distribution.

For an oblate grain with dynamic symmetry,

$q=1+\left(r_{2}-1\right) \sin ^{2} \gamma$

and $1 \leq q \leq r_{2}$. As seen in equation (11), the rotational kinetic energy (parametrized by $q$ for constant grain angular momentum $\boldsymbol{J})$ is unchanged if $\gamma \rightarrow \pi-\gamma$. In other words, $q$ does not depend on the sign of $\cos \gamma$. So to fully characterize the grain's rotational state, one must specify $\boldsymbol{J}, q$, and the $\operatorname{sign}$ of $\cos \gamma$ (which is equal to the sign of $\boldsymbol{J} \cdot \hat{\boldsymbol{a}}_{1}$ ). Weingartner \& Draine (2003) denoted the latter as the "flip state" of the grain. In order for a grain to flip, $q$ must increase to $r_{2}$, where $\gamma=\pi / 2$ and $\cos \gamma=0$, and then return to $q<r_{2}$, but with a change in $\operatorname{sign}$ of $\cos \gamma$ (i.e. in the opposite flip state).

Weingartner (2009) attempted to find the constant of integration in equation (10) by demanding that fluctuations cease to contribute to $S(q)$ in the limit that the dust temperature $T_{d} \rightarrow 0$. This must be true regardless of the form of $f(q)$. In order to be normalizable, $f(q)$ may diverge at any value $q_{0}$, but the divergence must be shallower than $f(q) \propto\left|q_{0}-q\right|^{-1}$. Defining

$b=\frac{J^{2}}{2 I_{1} k_{B} T_{d}}$

(with $k_{B}$ Boltzmann's constant), it must be the case that, for any $q$,

$\lim _{b^{-1} \rightarrow 0} \frac{\mathrm{d}[f(b, q) D(b, q)]}{\mathrm{d} q}=0$.

Weingartner (2009) claimed that the stronger condition

$\lim _{\left(b^{-1}, r_{2}-q\right) \rightarrow(0,0)} \frac{\mathrm{d}[f(b, q) D(b, q)]}{\mathrm{d} q}=0$

must be satisfied at $q=r_{2}$. This will only hold for all possible forms of $f(b, q)$ if $D(b, q)$ falls off at least as fast as $\left(r_{2}-q\right)^{2}$ near $q=r_{2}$, implying that both $D(q)$ and $\mathrm{d} D / \mathrm{d} q$ vanish at $q=r_{2}$. These results, along with the fact that $A(q)$ vanishes at $q=r_{2}$, make $q=r_{2}$ a natural boundary (Gardiner 2004). That is, assuming the condition in equation (14) is enforced, the grain cannot evolve to $q=r_{2}$ if it starts at $q \neq r_{2}$.

For non-symmetric grains with $I_{1}>I_{2}>I_{3}, 1 \leq q \leq r_{3}$, where $r_{3}=I_{1} / I_{3}$. Of course, $r_{3}>r_{2}$. As described in section 5 , for the grain to flip, it must start with $q<r_{2}$, evolve to $q>r_{2}$, and then evolve to $q<r_{2}$ again. If $q=r_{2}$ is a natural boundary, then, since the argument in the above paragraph did not make use of symmetry in any way, even non-symmetric grains cannot undergo thermal flipping.

However, we cannot see any reason why the stronger condition in equation (14) must be satisfied. Furthermore, nothing in the argument is restricted to $q=r_{2}$. It could be applied as well to any other value of $q$, making them all natural boundaries, which is absurd.

Even if $q=r_{2}$ is not a natural boundary, a symmetric grain never reaches $q=r_{2}$ for the same reason that it never reaches $q=1$, which is certainly not a natural boundary. The probability that $q$ evolves to exactly 1 or exactly $r_{2}$ is infinitesimal. Since $q$ must reach exactly $r_{2}$ for the grain to undergo a thermal flip, thermal flipping is not possible for symmetric grains. In non-symmetric grains, $q$ must simply evolve past $q=r_{2}$ in order to undergo a thermal flip (assuming $q=r_{2}$ is not a natural boundary, to be addressed in section 4 ).

The conclusion that thermal flipping is impossible for symmetric grains relies on the fact that the rotational kinetic energy $E$ is independent of the flip state and the assumption that the fluctuations are in $E$ (or equivalently $q$ for constant $\boldsymbol{J})$. Lazarian \& Roberge (1997) did not incorporate this assumption in their model of Barnett relaxation, enabling Lazarian \& Draine (1999a) to conclude that thermal flipping occurs for symmetric grains. When the evolution of the grain's rotational kinetic energy is affected by collisions with gas atoms, which also change the grain's angular momentum, this assumption no longer applies and flipping is possible. A detailed microphysical model of Barnett relaxation is needed to confirm or deny the above assumption and provide an unambiguous description of thermal flipping.

For oblate grains with dynamic symmetry, the equilibrium probability density is

$f_{\mathrm{TE}}(q) \propto \exp (-b q)\left(r_{2}-q\right)^{-1 / 2}$

(see, e.g., Weingartner 2009). Substituting for $A(q)$ and $f_{\mathrm{TE}}(q)$ from equations $(7)$ and (15) in equation (10) and integrating,

$b^{2} \tau_{\mathrm{Bar}} D(q, b)=[3+2 b(q-1)]\left(r_{2}-q\right)+C(b)\left(r_{2}-q\right)^{1 / 2} \exp (b q)-b^{-1 / 2}\left[3+2 b\left(r_{2}-1\right)\right]\left(r_{2}-q\right)^{1 / 2} F_{D}\left(\sqrt{b\left(r_{2}-q\right)}\right)$

where

$F_{D}(x)=\exp \left(-x^{2}\right) \int_{0}^{x} \exp \left(y^{2}\right) \mathrm{d} y$ 
is Dawson's integral and

$C(b)=b^{2} \exp (-b)\left(r_{2}-1\right)^{-1 / 2} \tau_{\mathrm{Bar}} D(q=1, b)-3 \exp (-b)\left(r_{2}-1\right)^{1 / 2}+b^{-1 / 2}\left[3+2 b\left(r_{2}-1\right)\right] \exp (-b) F_{D}\left(\sqrt{b\left(r_{2}-1\right)}\right)$.

When $r_{2}-q \ll 1$,

$b^{2} \tau_{\mathrm{Bar}} D(q, b) \approx C(b) \exp (b q)\left(r_{2}-q\right)^{1 / 2}+\frac{4}{3} b^{2}\left(r_{2}-1\right)\left(r_{2}-q\right)^{2}$.

Applying the condition in equation (14), Weingartner (2009) concluded that $C(b)=0$.

Dispensing with this condition, we find that $C(b)$ cannot be uniquely determined by examining the low-temperature limit (i.e. $b \rightarrow \infty$ ). Rather, if $C(b)$ is not identically zero, then it must fall off at least as fast as $b^{2} \exp \left(-b r_{2}\right)$ as $b \rightarrow \infty$. We have not been able to identify a general argument that can uniquely determine $C(b)$; perhaps this is only possible with a detailed microphysical model.

\section{BARNETT DISSIPATION IN NON-SYMMETRIC GRAINS}

\subsection{Euler's equations}

Consider a non-symmetric grain with principal moments of inertia $I_{1}>I_{2}>I_{3}$ and denote $r_{2}=I_{1} / I_{2}$ and $r_{3}=I_{1} / I_{3}$. When $1<q<r_{2}$, the solution of Euler's equations for the angular velocity $\omega$ is given in terms of Jacobi elliptic functions as

$\omega_{1}= \pm \frac{J}{I_{1}}\left(\frac{r_{3}-q}{r_{3}-1}\right)^{1 / 2} \operatorname{dn}\left(\omega_{\mathrm{rot}} t, k^{2}\right)$,

$\omega_{2}=-\frac{J}{I_{1}} r_{2}\left(\frac{q-1}{r_{2}-1}\right)^{1 / 2} \operatorname{sn}\left(\omega_{\text {rot }} t, k^{2}\right)$,

$\omega_{3}= \pm \frac{J}{I_{1}} r_{3}\left(\frac{q-1}{r_{3}-1}\right)^{1 / 2} \operatorname{cn}\left(\omega_{\mathrm{rot}} t, k^{2}\right)$

where

$k^{2}=\frac{\left(r_{3}-r_{2}\right)(q-1)}{\left(r_{2}-1\right)\left(r_{3}-q\right)}$

and

$\omega_{\text {rot }}=\frac{J}{I_{1}}\left[\left(r_{2}-1\right)\left(r_{3}-q\right)\right]^{1 / 2}$.

The grain is in the positive flip state with respect to $\hat{\boldsymbol{a}}_{1}$ (i.e. $\boldsymbol{J} \cdot \hat{\boldsymbol{a}}_{1}>0$ ) when the plus sign is chosen in both equations (20) and (22). It is in the negative flip state with respect to $\hat{\boldsymbol{a}}_{1}$ when the minus sign is chosen in both of those cases. We adopt the same conventions for the Jacobi elliptic functions as Weingartner \& Draine (2003).

When $r_{2}<q<r_{3}$,

$\omega_{1}= \pm \frac{J}{I_{1}}\left(\frac{r_{3}-q}{r_{3}-1}\right)^{1 / 2} \operatorname{cn}\left(\omega_{\text {rot }} t, k^{-2}\right)$

$\omega_{2}=-\frac{J}{I_{1}} r_{2}\left(\frac{r_{3}-q}{r_{3}-r_{2}}\right)^{1 / 2} \operatorname{sn}\left(\omega_{\text {rot }} t, k^{-2}\right)$

$\omega_{3}= \pm \frac{J}{I_{1}} r_{3}\left(\frac{q-1}{r_{3}-1}\right)^{1 / 2} \mathrm{dn}\left(\omega_{\mathrm{rot}} t, k^{-2}\right)$,

with

$\omega_{\text {rot }}=\frac{J}{I_{1}}\left[\left(r_{3}-r_{2}\right)(q-1)\right]^{1 / 2}$.

The grain is in the positive flip state with respect to $\hat{\boldsymbol{a}}_{3}$ (i.e. $\boldsymbol{J} \cdot \hat{\boldsymbol{a}}_{3}>0$ ) when the plus sign is chosen in both equations $(25)$ and (27). It is in the negative flip state with respect to $\hat{\boldsymbol{a}}_{3}$ when the minus sign is chosen in both of those cases.

As for symmetric grains, the Barnett equivalent field $\boldsymbol{B}_{\mathrm{BE}}=\omega / \gamma_{g}$.

\subsection{Modified Bloch equations}

Since the Bloch equations do not accomodate dissipation in the limit of zero biasing field, we adopt the modified Bloch equations of Wangsness (1956). These are

$\frac{\mathrm{d} M_{x}}{\mathrm{~d} t}=\gamma_{g}\left(M_{y} B_{z}-M_{z} B_{y}\right)-\frac{M_{x}-\chi_{0} B_{x}}{T_{2}}$ 
$\frac{\mathrm{d} M_{y}}{\mathrm{~d} t}=\gamma_{g}\left(M_{z} B_{x}-M_{x} B_{z}\right)-\frac{M_{y}-\chi_{0} B_{y}}{T_{2}}$,

$\frac{\mathrm{d} M_{z}}{\mathrm{~d} t}=\gamma_{g}\left(M_{x} B_{y}-M_{y} B_{x}\right)-\frac{M_{z}-\chi_{0} B_{z}}{T_{1}}$,

where $M$ is the magnetization, $T_{1}$ is the spin-lattice relaxation time, and the other quantities were introduced in section 2 . In contrast to typical magnetic resonance experiments, where $B_{z}$ is constant, all three Barnett equivalent fields are time-variable for the case of a nonsymmetric grain. It is not clear how to further modify the Bloch equations for this case, so we will simply set $T_{1}=T_{2}$.

Taking $(\hat{\boldsymbol{x}}, \hat{\boldsymbol{y}}, \hat{\boldsymbol{z}})$ in the modified Bloch equations to lie along $\left(\hat{\boldsymbol{a}}_{2}, \hat{\boldsymbol{a}}_{3}, \hat{\boldsymbol{a}}_{1}\right)$ and introducing dimensionless variables $t^{\prime}=t / T_{2}$,

$T_{2}^{\prime}=\omega_{\text {rot }} T_{2}$,

$\boldsymbol{B}^{\prime}=\frac{\gamma_{g} \boldsymbol{B}}{\omega_{\mathrm{rot}}^{2} T_{2}}$,

and

$M^{\prime}=\frac{\gamma_{g} M}{\chi_{0} \omega_{\text {rot }}}$,

the modified Bloch equations become

$\frac{\mathrm{d} M_{2}^{\prime}}{\mathrm{d} t^{\prime}}=\left(T_{2}^{\prime}\right)^{2}\left(M_{3}^{\prime} B_{1}^{\prime}-M_{1}^{\prime} B_{3}^{\prime}\right)-M_{2}^{\prime}+T_{2}^{\prime} B_{2}^{\prime}$,

$\frac{\mathrm{d} M_{3}^{\prime}}{\mathrm{d} t^{\prime}}=\left(T_{2}^{\prime}\right)^{2}\left(M_{1}^{\prime} B_{2}^{\prime}-M_{2}^{\prime} B_{1}^{\prime}\right)-M_{3}^{\prime}+T_{2}^{\prime} B_{3}^{\prime}$,

$\frac{\mathrm{d} M_{1}^{\prime}}{\mathrm{d} t^{\prime}}=\left(T_{2}^{\prime}\right)^{2}\left(M_{2}^{\prime} B_{3}^{\prime}-M_{3}^{\prime} B_{2}^{\prime}\right)-M_{1}^{\prime}+T_{2}^{\prime} B_{1}^{\prime}$.

We will consider the low-frequency limit, with $T_{2}^{\prime} \ll 1$.

3.3 The case that $1<q<r_{2}$

From equations (20)-(22), when $1<q<r_{2}$,

$B_{1}^{\prime}=\left(T_{2}^{\prime}\right)^{-1} c_{1} \operatorname{dn}\left(T_{2}^{\prime} t^{\prime}, k^{2}\right)$,

$B_{2}^{\prime}=\left(T_{2}^{\prime}\right)^{-1} c_{2} \operatorname{sn}\left(T_{2}^{\prime} t^{\prime}, k^{2}\right)$,

$B_{3}^{\prime}=\left(T_{2}^{\prime}\right)^{-1} c_{3} \operatorname{cn}\left(T_{2}^{\prime} t^{\prime}, k^{2}\right)$,

with

$c_{1}=\left[\left(r_{2}-1\right)\left(r_{3}-1\right)\right]^{-1 / 2}$,

$c_{2}=-\frac{r_{2}}{r_{2}-1}\left(\frac{q-1}{r_{3}-q}\right)^{1 / 2}$,

$c_{3}=r_{3}\left[\frac{q-1}{\left(r_{2}-1\right)\left(r_{3}-1\right)\left(r_{3}-q\right)}\right]^{1 / 2}$.

The solution to the modified Bloch equations is

$M_{1}^{\prime}=c_{1} \operatorname{dn}\left(T_{2}^{\prime} t^{\prime}, k^{2}\right)+T_{2}^{\prime} k^{2} c_{1} \operatorname{sn}\left(T_{2}^{\prime} t^{\prime}, k^{2}\right) \operatorname{cn}\left(T_{2}^{\prime} t^{\prime}, k^{2}\right)+\left(T_{2}^{\prime}\right)^{2} \operatorname{dn}\left(T_{2}^{\prime} t^{\prime}, k^{2}\right)\left\{k^{2} c_{1}\left[2 \operatorname{sn}^{2}\left(T_{2}^{\prime} t^{\prime}, k^{2}\right)-1\right]-c_{2} c_{3}\right\}+O\left[\left(T_{2}^{\prime}\right)^{3}\right]$, 
$M_{2}^{\prime}=c_{2} \operatorname{sn}\left(T_{2}^{\prime} t^{\prime}, k^{2}\right)-T_{2}^{\prime} c_{2} \operatorname{cn}\left(T_{2}^{\prime} t^{\prime}, k^{2}\right) \operatorname{dn}\left(T_{2}^{\prime} t^{\prime}, k^{2}\right)$

$$
+\left(T_{2}^{\prime}\right)^{2} \operatorname{sn}\left(T_{2}^{\prime} t^{\prime}, k^{2}\right)\left\{\left(1-k^{2}\right) c_{1} c_{3}-c_{2}\left[2 \mathrm{dn}^{2}\left(T_{2}^{\prime} t^{\prime}, k^{2}\right)-\left(1-k^{2}\right)\right]\right\}+O\left[\left(T_{2}^{\prime}\right)^{3}\right],
$$

$M_{3}^{\prime}=c_{3} \operatorname{cn}\left(T_{2}^{\prime} t^{\prime}, k^{2}\right)+T_{2}^{\prime} c_{3} \operatorname{sn}\left(T_{2}^{\prime} t^{\prime}, k^{2}\right) \operatorname{dn}\left(T_{2}^{\prime} t^{\prime}, k^{2}\right)+\left(T_{2}^{\prime}\right)^{2} \operatorname{cn}\left(T_{2}^{\prime} t^{\prime}, k^{2}\right)\left\{c_{1} c_{2}+c_{3}\left[1-2 \operatorname{dn}^{2}\left(T_{2}^{\prime} t^{\prime}, k^{2}\right)\right]\right\}+O\left[\left(T_{2}^{\prime}\right)^{3}\right]$,

where $O\left[\left(T_{2}^{\prime}\right)^{3}\right]$ denotes terms of order $\left(T_{2}^{\prime}\right)^{3}$ and higher.

The instantaneous absorbed power per unit volume is $B \cdot \mathrm{d} \boldsymbol{M} / \mathrm{d} t$. The period of the motion is $4 \omega_{\text {rot }}^{-1} K\left(k^{2}\right)$, where $K\left(k^{2}\right)$ is the complete elliptic integral of the first kind,

$K\left(k^{2}\right)=\int_{0}^{\pi / 2} \mathrm{~d} \theta\left(1-k^{2} \sin ^{2} \theta\right)^{-1 / 2}$.

Thus, the grain's rotational kinetic energy is dissipated at rate

$\frac{\mathrm{d} E}{\mathrm{~d} t}=-\frac{V \omega_{\mathrm{rot}}}{4 K\left(k^{2}\right)} \int_{0}^{4 \omega_{\mathrm{rot}}^{-1} K\left(k^{2}\right)} \mathrm{d} t \boldsymbol{B} \cdot \frac{\mathrm{d} \boldsymbol{M}}{\mathrm{d} t}=-\frac{\chi_{0} V T_{2} \omega_{\mathrm{rot}}^{4}}{4 K\left(k^{2}\right) \gamma_{g}^{2}} \int_{0}^{4 K\left(k^{2}\right) / T_{2}^{\prime}} \mathrm{d} t^{\prime} \boldsymbol{B}^{\prime} \cdot \frac{\mathrm{d} \boldsymbol{M}^{\prime}}{\mathrm{d} t^{\prime}}$

where $V$ is the grain volume. To first order in $T_{2}^{\prime}$,

$$
\begin{aligned}
B^{\prime} \cdot \frac{d \boldsymbol{M}^{\prime}}{d t^{\prime}}=\left(c_{2}^{2}-k^{2} c_{1}^{2}-c_{3}^{2}\right) \operatorname{sn}\left(T_{2}^{\prime} t^{\prime}, k^{2}\right) \operatorname{cn}\left(T_{2}^{\prime} t^{\prime}, k^{2}\right) \operatorname{dn}\left(T_{2}^{\prime} t^{\prime}, k^{2}\right)+T_{2}^{\prime}\left[k^{2}\left(c_{2}^{2}-c_{3}^{2}\right) \operatorname{sn}^{2}\left(T_{2}^{\prime} t^{\prime}, k^{2}\right) \mathrm{cn}^{2}\left(T_{2}^{\prime} t^{\prime}, k^{2}\right)\right. \\
\left.+\left(c_{2}^{2}-k^{2} c_{1}^{2}\right) \operatorname{sn}^{2}\left(T_{2}^{\prime} t^{\prime}, k^{2}\right) \operatorname{dn}^{2}\left(T_{2}^{\prime} t^{\prime}, k^{2}\right)+\left(k^{2} c_{1}^{2}+c_{3}^{2}\right) \operatorname{cn}^{2}\left(T_{2}^{\prime} t^{\prime}, k^{2}\right) \mathrm{dn}^{2}\left(T_{2}^{\prime} t^{\prime}, k^{2}\right)\right] .
\end{aligned}
$$

The zeroth-order term integrates to zero identically. The first-order term then yields

$\int_{0}^{4 K\left(k^{2}\right) / T_{2}^{\prime}} d t^{\prime} \boldsymbol{B}^{\prime} \cdot \frac{d \boldsymbol{M}^{\prime}}{d t^{\prime}}=\frac{4\left\{z_{1}\left[E\left(k^{2}\right)+\left(k^{2}-1\right) K\left(k^{2}\right)\right]+k^{2} z_{2} E\left(k^{2}\right)\right\}(q-1)}{3 k^{2}\left(r_{2}-1\right)^{2}\left(r_{3}-1\right)\left(r_{3}-q\right)}+O\left(T_{2}^{\prime}\right)$

where $E\left(k^{2}\right)$ is the complete elliptic integral of the second kind,

$E\left(k^{2}\right)=\int_{0}^{\pi / 2} \mathrm{~d} \theta\left(1-k^{2} \sin ^{2} \theta\right)^{1 / 2}$,

$z_{1}=2\left(r_{3}-r_{2}\right)-r_{3}^{2}\left(r_{2}-1\right)+r_{2}^{2}\left(r_{3}-1\right)$

and

$z_{2}=-\left(r_{3}-r_{2}\right)+2 r_{3}^{2}\left(r_{2}-1\right)+r_{2}^{2}\left(r_{3}-1\right)$.

Thus,

$A(q)=\frac{\mathrm{d} q}{\mathrm{~d} t}=-\tau_{\text {int }}^{-1} \frac{\left\{z_{1}\left[P\left(k^{2}\right)+k^{2}-1\right]+k^{2} z_{2} P\left(k^{2}\right)\right\}(q-1)\left(r_{3}-q\right)}{3 k^{2}\left(r_{3}-1\right)}$

where $P\left(k^{2}\right)=E\left(k^{2}\right) / K\left(k^{2}\right)$ and

$\tau_{\text {int }}=\frac{\gamma_{g}^{2} I_{1}^{3}}{2 \chi_{0} V T_{2} J^{2}}$.

In the limit $r_{3} \rightarrow r_{2}, k^{2} \rightarrow 0, z_{1} \rightarrow 0, z_{2} \rightarrow 3 r_{2}^{2}\left(r_{2}-1\right)$, and

$\frac{z_{1}\left[P\left(k^{2}\right)+k^{2}-1\right]+k^{2} z_{2} P\left(k^{2}\right)}{k^{2}} \rightarrow z_{2}$.

Thus,

$A(q) \rightarrow-\frac{2 \chi_{0} V T_{2} J^{2}}{\gamma_{g}^{2} I_{1} I_{2}^{2}}(q-1)\left(r_{2}-q\right)$

which reproduces equation (7) for a symmetric grain when $\omega_{\text {rot }} T_{2} \ll 1$.

In the limit $q \rightarrow 1$,

$A(q) \rightarrow-\tau_{\text {int }}^{-1} \frac{r_{3}^{2}\left(r_{2}-1\right)+r_{2}^{2}\left(r_{3}-1\right)}{2}(q-1)$.

Note that this expression also agrees with the result for a symmetric grain when $r_{2}=r_{3}$.

As expected, $A(q) \rightarrow 0$ when $q \rightarrow r_{2}$, though this is not apparent from inspection of equation (55). In this limit,

$A(q) \rightarrow-\tau_{\text {int }}^{-1} \frac{2\left(r_{2}-1\right)\left(r_{3}-r_{2}\right)}{3\left(r_{3}-1\right)}\left[r_{3}-r_{2}+r_{3}^{2}\left(r_{2}-1\right)+2 r_{2}^{2}\left(r_{3}-1\right)\right]\left\{\ln \left[\frac{\left(r_{2}-1\right)\left(r_{3}-r_{2}\right)}{\left(r_{3}-1\right)\left|r_{2}-q\right|}\right]\right\}^{-1}$.

Thus, $A(q)$ falls off much faster with $r_{2}-q$ than for a symmetric grain as $q \rightarrow r_{2}$. With the absolute value of $r_{2}-q$ in equation (60), the expression is correct in the $q \rightarrow r_{2}$ for both $q<r_{2}$ and $q>r_{2}$. 
3.4 The case that $r_{2}<q<r_{3}$

When $r_{2}<q<r_{3}$, a derivation analogous to that above yields

$A(q)=-\tau_{\text {int }}^{-1} \frac{\left\{z_{2}\left[P\left(k^{-2}\right)+k^{-2}-1\right]+k^{-2} z_{1} P\left(k^{-2}\right)\right\}(q-1)\left(r_{3}-q\right)}{3 k^{-2}\left(r_{3}-1\right)}$.

In the limit $q \rightarrow r_{3}$,

$A(q) \rightarrow-\tau_{\text {int }}^{-1} \frac{r_{3}-r_{2}+r_{2}^{2}\left(r_{3}-1\right)}{2}\left(r_{3}-q\right)$.

\section{THE DIFFUSION COEFFICIENT FOR NON-SYMMETRIC GRAINS}

As for symmetric grains, we use equation (10) to find the diffusion coefficient. The drift coefficient $A(q)$ is given by equations (55) and (61) for the cases that $q<r_{2}$ and $q>r_{2}$, respectively. For later convenience, define $A_{1}(q)=-\tau_{\text {int }} A(q)$.

The thermal-equilibrium distribution function is

$f_{\mathrm{TE}}(q) \propto \exp (-b q) s^{\prime}(q)$

where

$s(q)=1-\frac{2}{\pi} \int_{0}^{\alpha_{\max }} \mathrm{d} \alpha\left[\frac{r_{2}-q+\left(r_{3}-r_{2}\right) \cos ^{2} \alpha}{r_{2}-1+\left(r_{3}-r_{2}\right) \cos ^{2} \alpha}\right]^{1 / 2}$,

$\alpha_{\max }=\left\{\begin{array}{ll}\pi / 2 & , q \leq r_{2} \\ \cos ^{-1}\left[\frac{q-r_{2}}{r_{3}-r_{2}}\right]^{1 / 2} & , q>r_{2}\end{array}\right.$,

and $s^{\prime}(q)=\mathrm{d} s / \mathrm{d} q$ (Weingartner 2009). Thus,

$\begin{aligned} s^{\prime}(q) & =\pi^{-1} \int_{0}^{\alpha_{\max }} \mathrm{d} \alpha\left\{\left[r_{2}-1+\left(r_{3}-r_{2}\right) \cos ^{2} \alpha\right]\left[r_{2}-q+\left(r_{3}-r_{2}\right) \cos ^{2} \alpha\right]\right\}^{-1 / 2} \\ & =\pi^{-1} \int_{0}^{\alpha_{\max }} \mathrm{d} \alpha\left\{\left[r_{3}-1-\left(r_{3}-r_{2}\right) \sin ^{2} \alpha\right]\left[r_{3}-q-\left(r_{3}-r_{2}\right) \sin ^{2} \alpha\right]\right\}^{-1 / 2}\end{aligned}$

except when $q=r_{3}$, for which

$s^{\prime}\left(r_{3}\right)=\frac{1}{2}\left[\left(r_{3}-r_{2}\right)\left(r_{3}-1\right)\right]^{-1 / 2}$.

When $q=1$,

$s^{\prime}(1)=\frac{1}{2}\left[\left(r_{2}-1\right)\left(r_{3}-1\right)\right]^{-1 / 2}$.

After slight manipulation, the expression in equation (67) is of the form of integral 2.616\#1 in Gradshteyn \& Ryzhik (2015), yielding

$s^{\prime}(q)=\pi^{-1}\left[\left(r_{2}-1\right)\left(r_{3}-q\right)\right]^{-1 / 2} K\left(k^{2}\right)$

when $q<r_{2}$. Since $K\left(k^{2}\right) \rightarrow \pi / 2$ as $k \rightarrow 0, s^{\prime}(q) \rightarrow \frac{1}{2}\left[\left(r_{2}-1\right)\left(r_{2}-q\right)\right]^{-1 / 2}$ as $r_{3} \rightarrow r_{2}$, reproducing the result for a symmetric grain (see eq. 13 in Weingartner 2009). A change of variables $x=\left(r_{3}-r_{2}\right) \sin ^{2} \alpha$ puts the expression in equation (67) into the form of integral 3.147\#3 in Gradshteyn \& Ryzhik (2015), yielding

$s^{\prime}(q)=\pi^{-1}\left[\left(r_{3}-r_{2}\right)(q-1)\right]^{-1 / 2} K\left(k^{-2}\right)$

when $q>r_{2}$. As for a symmetric grain, $s^{\prime}(q) \rightarrow \infty$ as $q \rightarrow r_{2}$.

Defining $G(q)=A_{1}(q) s^{\prime}(q)$, equation (10) becomes

$\tau_{\text {int }} D(q, b)=\frac{\exp (b q)}{s^{\prime}(q)}\left[\tau_{\text {int }} D(1, b) \exp (-b) s^{\prime}(1)-2 \int_{1}^{q} G\left(q^{\prime}\right) \exp \left(-b q^{\prime}\right) \mathrm{d} q^{\prime}\right]$.

From equations (55) and (70),

$G(q)=\frac{\left(r_{2}-1\right)^{1 / 2}\left(r_{3}-q\right)^{3 / 2}\left[\left(z_{1}+k^{2} z_{2}\right) E\left(k^{2}\right)-z_{1}\left(1-k^{2}\right) K\left(k^{2}\right)\right]}{3 \pi\left(r_{3}-1\right)\left(r_{3}-r_{2}\right)}$

when $q<r_{2}$. Similarly, from equations (61) and (71),

$G(q)=\frac{\left(r_{3}-r_{2}\right)^{1 / 2}(q-1)^{3 / 2}\left[\left(z_{2}+k^{-2} z_{1}\right) E\left(k^{-2}\right)-z_{2}\left(1-k^{-2}\right) K\left(k^{-2}\right)\right]}{3 \pi\left(r_{3}-1\right)\left(r_{2}-1\right)}$

when $q>r_{2}$. 


\subsection{The boundary at $q=r_{2}$}

With the above results, we are now prepared to demonstrate that the boundary at $q=r_{2}$ is not a natural boundary. Recall that $A(q), D(q)$, and $\mathrm{d} D / \mathrm{d} q$ must all vanish at $q=r_{2}$ in order for it to be a natural boundary. Of course, $A(q)$ vanishes at $q=r_{2}$. As noted above, $s^{\prime}(q) \rightarrow \infty$ as $q \rightarrow r_{2}$. Since the integral in equation (72) does not diverge, $D(q)$ also vanishes at $q=r_{2}$. To see that the integral does not diverge, it is sufficient to note that the function $G(q)$ does not diverge anywhere. This function vanishes as $q \rightarrow 1$ and $q \rightarrow r_{2}$ and

$G(q) \rightarrow \frac{\left[\left(r_{2}-1\right)\left(r_{3}-r_{2}\right)\right]^{1 / 2}\left(z_{1}+z_{2}\right)}{3 \pi\left(r_{3}-1\right)}$

as $q \rightarrow r_{2}$ (from both below and above). As $q \rightarrow r_{2}, k^{2} \rightarrow 1, E\left(k^{2}\right) \rightarrow 1$, and $K\left(k^{2}\right) \rightarrow-\frac{1}{2} \ln \left(1-k^{2}\right)$. Thus, the term $\left(1-k^{2}\right) K\left(k^{2}\right)$ in $G(q)$ does not diverge; rather, it vanishes as $q \rightarrow r_{2}$.

The derivative

$\frac{\mathrm{d}\left(\tau_{\text {int }} D\right)}{\mathrm{d} q}\left(q=r_{2}\right)=-\frac{2 G\left(r_{2}\right)}{s^{\prime}\left(r_{2}\right)}+\frac{b \exp \left(b r_{2}\right) H\left(r_{2}\right)}{s^{\prime}\left(r_{2}\right)}-\frac{\exp \left(b r_{2}\right) H\left(r_{2}\right) s^{\prime \prime}\left(r_{2}\right)}{\left[s^{\prime}\left(r_{2}\right)\right]^{2}}$

where $H(q)$ is the quantity in square brackets in equation (72),

$H(q)=\tau_{\text {int }} D(1, b) \exp (-b) s^{\prime}(1)-2 \int_{1}^{q} G\left(q^{\prime}\right) \exp \left(-b q^{\prime}\right) d q^{\prime}$.

Since $s^{\prime}(q)$ diverges at $q=r_{2}$ and $G(q)$ does not, the first term on the right-hand side in equation $(76)$ vanishes. Since $D(q)$ must be non-negative for all $q$, so must $H(q)$. Since $G(q)$ is non-negative, $H(q)$ can, at most, vanish only at $q=r_{3}$. Thus, the numerator in the second term on the right-hand side in equation (76) is non-zero but does not diverge, implying that the second term vanishes. From equations (70) and (71) and the relation

$\frac{\mathrm{d} K\left(k^{2}\right)}{\mathrm{d} k^{2}}=\frac{E\left(k^{2}\right)-\left(1-k^{2}\right) K\left(k^{2}\right)}{2 k^{2}\left(1-k^{2}\right)}$

$s^{\prime \prime}(q) \rightarrow \pm \frac{r_{3}-1}{2 \pi\left[\left(r_{2}-1\right)\left(r_{3}-r_{2}\right)\right]^{3 / 2}} \frac{1}{1-k^{ \pm 2}}$

as $q \rightarrow r_{2}$; the $+(-)$ sign applies when $q<r_{2}\left(q>r_{2}\right)$. Thus, the final term on the right-hand side in equation (76) approaches $\mp \infty$ as $q \rightarrow r_{2}$ from below (minus sign) or above. That is, $\mathrm{d} D / \mathrm{d} q$ diverges at $q=r_{2}$ and this point is not a natural boundary.

\subsection{The constant of integration}

As for symmetric grains, we demand that $D(q, b) \rightarrow 0$ as $b \rightarrow 0$. Thus, it must be that $H\left(q=r_{3}, b\right)$ falls off at least as rapidly as $\exp \left(-b r_{3}\right)$. Of course, $H\left(q=r_{3}, b\right) \equiv 0$ satisfies this condition. This choice corresponds to that made by Weingartner (2009) for symmetric grains, i.e. $C(b) \equiv 0$. As $q \rightarrow r_{3}$,

$s^{\prime \prime}(q) \rightarrow-\frac{1}{4}\left[\left(r_{3}-r_{2}\right)\left(r_{3}-1\right)\right]^{-1 / 2}\left(r_{2}-1\right)^{-1}$.

Since $s^{\prime}\left(r_{3}\right)$ and $s^{\prime \prime}\left(r_{3}\right)$ are both non-zero and non-divergent and $G\left(r_{3}\right)=0$, if $H\left(r_{3}\right)=0$ then $q=r_{3}$ is a natural boundary.

\section{SIMULATIONS OF BARNETT RELAXATION FOR NON-SYMMETRIC GRAINS}

Lacking a first-principles model of Barnett relaxation, we will adopt $H\left(r_{3}\right) \equiv 0$ for simplicity. Equation (72) becomes

$\tau_{\mathrm{int}} D(q, b)=\frac{2 \exp (b q)}{s^{\prime}(q)} \int_{q}^{r_{3}} G\left(q^{\prime}\right) \exp \left(-b q^{\prime}\right) \mathrm{d} q^{\prime}$

From the definition of the inertia tensor, $r_{3}$ can be arbitrarily large but it must be that $r_{2} \leq r_{3} /\left(r_{3}-1\right)$. In order to check the thermal flipping rate, we ran simulations of Barnett relaxation for three grain shapes. Shapes 1,2 , and 3 have $\left(r_{2}, r_{3}\right)=$ $(1.3,1.5),(1.49,1.5)$, and $(1.01,1.5)$, respectively.

The Langevin equation is

$\mathrm{d} q=A(q) \mathrm{d} t+\sqrt{D(q)} \mathrm{d} w$

where $\mathrm{d} t$ is a time step and $\mathrm{d} w$ is a Gaussian random variable with variance $\mathrm{d} t$. Adopting dimensionless quantities $\mathrm{d} t^{\prime}=\mathrm{d} t / \tau_{\text {int }}$, $\mathrm{d} w^{\prime}=\mathrm{d} w / \sqrt{\tau_{\text {int }}}, B_{1}(q)=\sqrt{\tau_{\text {int }} D(q)}$, and recalling the definition $A_{1}(q)=-\tau_{\text {int }} A(q)$, the Langevin equation becomes

$\mathrm{d} q=-A_{1}(q) \mathrm{d} t^{\prime}+B_{1}(q) \mathrm{d} w^{\prime}$.

For each grain shape, we consider $b=1.0,5.0,10.0$, and 20.0. For each simulation, we first construct interpolation tables for $A_{1}(q)$ and $B_{1}(q)$. As seen in Fig. 1, these functions drop steeply to zero as $q \rightarrow r_{2}$, where flipping occurs. Thus, we tabulate in parameter $\log \left(r_{2}-q\right)$ or $\log \left(q-r_{2}\right)$ when $q<r_{2}$ or $q>r_{2}$, respectively, rather than in $q$. The tables contain values for $2 \times 10^{4}$ 

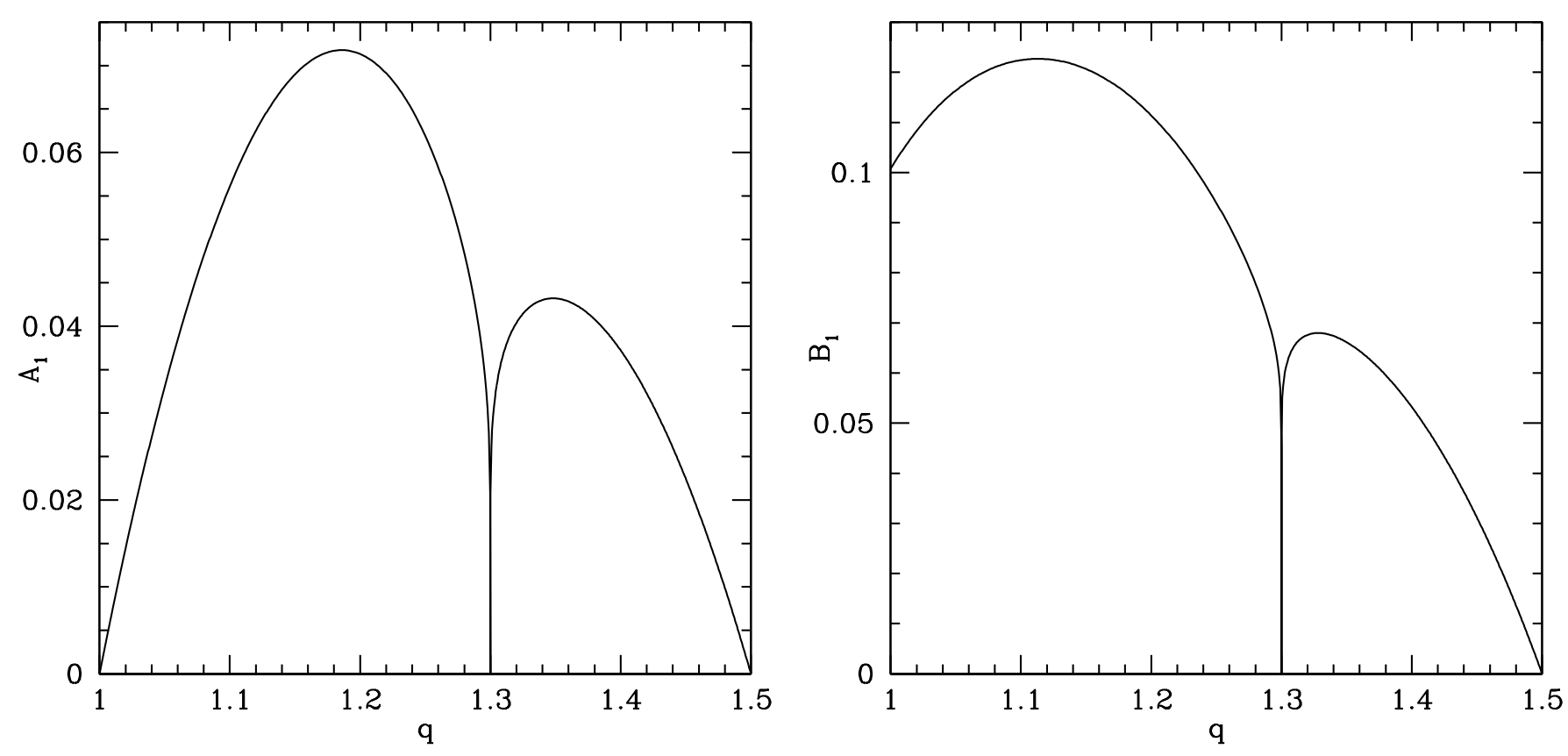

Figure 1. Left: $A_{1}(q)=-\tau_{\text {int }} A(q)$, where $A(q)$ is the drift coefficient, for shape $1\left(r_{2}=1.3, r_{3}=1.5\right)$. Right: $B_{1}(q)=\sqrt{\tau_{\text {int }} D(q)}$, where $D(q)$ is the diffusion coefficient, for shape 1 and $b=10.0$.

values of $q$ (half with $q<r_{2}$ and half with $q>r_{2}$ ) computed using MATHEMATICA. We also ran a simulation with $2 \times 10^{3}$ values of $q$ in the interpolation tables and found that the results were not significantly affected.

The simulations adopt a step size $\mathrm{d} t^{\prime}=10^{-5}$. We also ran a simulation with step size $\mathrm{d} t^{\prime}=10^{-6}$ and found that the average flip time was not substantially affected. If a step causes the grain to overstep a boundary (i.e. $q<1$ or $q>r_{3}$ ), then $\mathrm{d} t^{\prime}$ is reduced by a factor of 10 and the step is attempted again. Random numbers and the Gaussian random variables $\mathrm{d} w$ are computed using modified versions of the routines RAN2 and GASDEv from Press et al. (1992). As seen in Fig. 4 of Weingartner \& Draine (2003), a grain with $q$ slightly larger than $r_{2}$ can naturally transition into either the positive or negative flip state with respect to $\hat{\boldsymbol{a}}_{1}$ if $q$ changes to a value somewhat less than $r_{2}$. Which flip state results depends on the phase of the grain rotation at the moment when $q$ crosses $r_{2}$. Half of the total phase corresponds to a transition to the positive flip state and the other half to the negative flip state. Thus, whenever $q$ crosses $r_{2}$ from above, the flip state with respect to $\hat{\boldsymbol{a}}_{1}$ is chosen randomly, with equal probability for the positive and negative flip states. (We do not bother to track the flip states with respect to $\hat{\boldsymbol{a}}_{3}$ when $q>r_{2}$.)

Fig. 2 shows the results of a simulation for shape 1 with $b=10.0$. A flip occurs when the grain starts in one flip state with respect to $\hat{\boldsymbol{a}}_{1}$ with $q<r_{2}$, evolves to $q>r_{2}$, and evolves back to $q<r_{2}$ in the opposite flip state with respect to $\hat{\boldsymbol{a}}_{1}$. The right panel of Fig. 2 zooms in on the first opportunity for a flip to occur, when $t^{\prime} \approx 7$. Not surprisingly, there are multiple crossings of $q=r_{2}$ in rapid succession, with $q$ remaining near $r_{2}$. It would not be sensible to count each of these crossings as a potential flip. Instead, we adopt the following criterion for a genuine flip to occur: the grain must cross $q=r_{2}$ from below and later (perhaps after multiple crossing of $\left.q=r_{2}\right)$ return to $q=\frac{1}{2}\left(1+r_{2}\right)$ in the opposite flip state.

We ran long simulations to find the average time between flips, $\tau_{\text {flip }}$, for the three shapes and four values of $b$ indicated above. The results for $\tau_{\text {flip }}^{\prime}=\tau_{\text {flip }} / \tau_{\text {int }}$ are summarized in Table 1. Each shape exhibits rapid thermal flipping for sufficiently low $b$. As the grain shape approaches that of an oblate symmetric grain $\left(r_{2}=r_{3}\right)$, the flipping rate decreases. This is as expected, since we chose the constant of integration for the diffusion coefficient to be consistent with the choice in Weingartner (2009). For shape 3, with $r_{2}$ close to 1 and $r_{3}$ considerably larger, the flipping rate is relatively fast. In this case, so long as $b$ is not too large, the grain spends much or most of the time with $q>r_{2}$, i.e. in a flip state with respect to $\hat{\boldsymbol{a}}_{3}$.

For comparison, the last column in Table 1 is $\tau_{\text {flip }}^{\prime}(\operatorname{LD} 99)=\{\exp [-(b-1) / 2]\}^{-1}$, the approximate expression for the flip time from Lazarian \& Draine (1999a).

\section{SUMMARY}

The following are our principal results.

1. We revisited Barnett relaxation in grains with dynamic symmetry, concluding that thermal flipping (i.e. flipping 

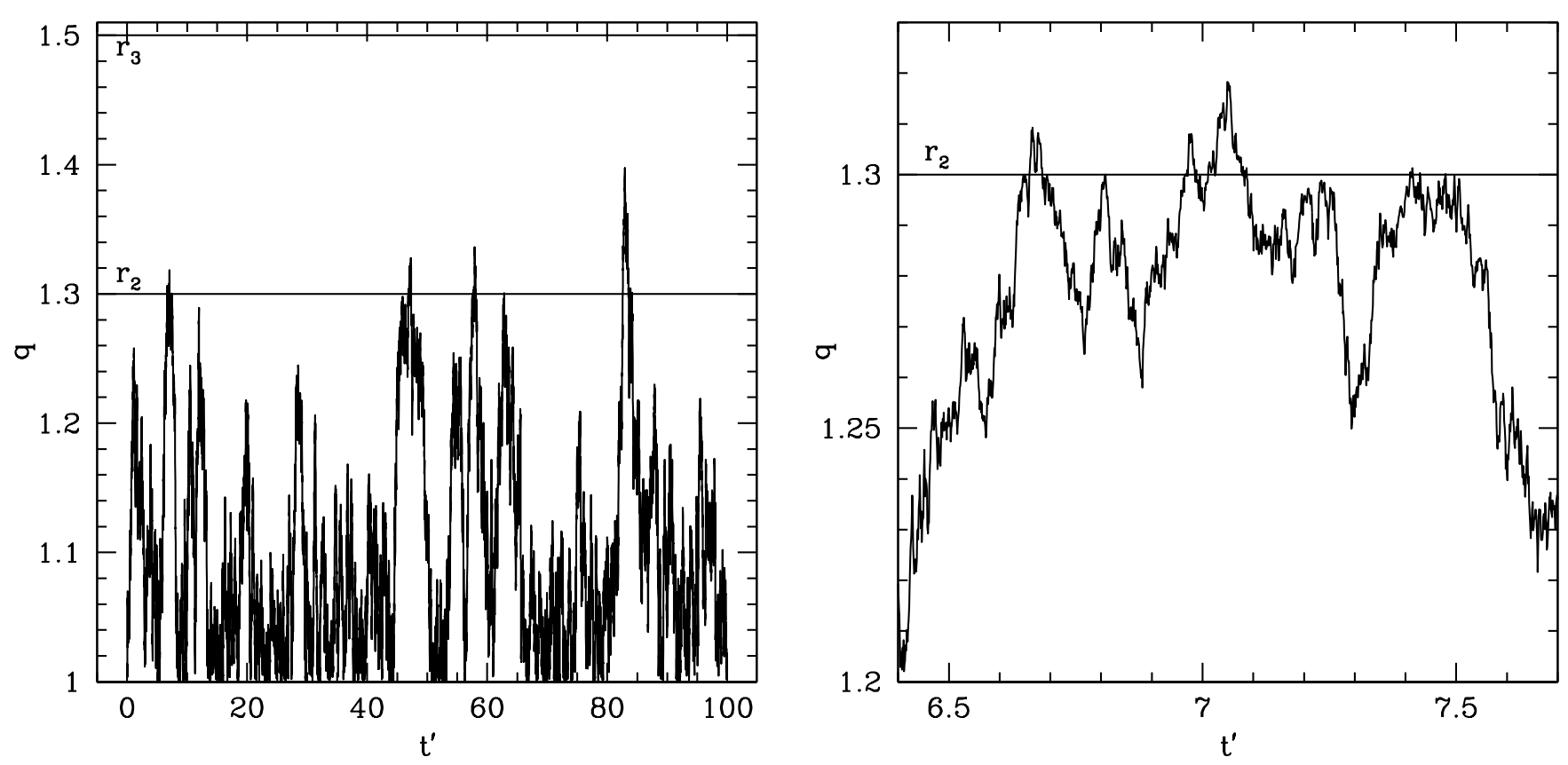

Figure 2. Left: Simulation of Barnett relaxation ( $q$ versus $\left.t^{\prime}=t / \tau_{\text {int }}\right)$ for shape $1\left(r_{2}=1.3, r_{3}=1.5\right)$ with $b=10$. Right: A zoom-in on the region around a potential flip.

Table 1. Average time between flips in stochastic simulations

\begin{tabular}{lllll}
\hline Shape & $b$ & $\tau_{\text {flip }}^{\prime}$ & Number of flips & $\tau_{\text {flip }}^{\prime}($ LD99) \\
\hline 1 & 1.0 & 13.96 & 286438 & 1.0 \\
1 & 5.0 & 21.27 & 2960253 & 7.4 \\
1 & 10.0 & 48.39 & 289085 & 90 \\
1 & 20.0 & 448.3 & 187133 & $1.4 \times 10^{4}$ \\
2 & 1.0 & 109.4 & 54834 & 1.0 \\
2 & 5.0 & 259.4 & 46207 & 7.4 \\
2 & 10.0 & 1272 & 49398 & 90 \\
2 & 20.0 & $6.942 \times 10^{4}$ & 1949 & $1.4 \times 10^{4}$ \\
3 & 1.0 & 0.3350 & 17896669 & 1.0 \\
3 & 5.0 & 0.4254 & 28184360 & 7.4 \\
3 & 10.0 & 0.5869 & 71503365 & 90 \\
3 & 20.0 & 0.9727 & 86290047 & $1.4 \times 10^{4}$ \\
\hline
\end{tabular}

induced by internal relaxation) does not occur. We do not agree with Weingartner (2009) that $q=r_{2}$ must be a natural boundary, although it could be depending on the value of the unknown constant of integration in equation (10). In any case, the probability that a grain reaches exactly $q=r_{2}$, required for a flip to occur, is infinitesimal.

2. We derived expressions for the Barnett dissipation rate for grains lacking dynamic symmetry (equations 55 and 61) in the limit of low rotational frequency (i.e. the spin-spin relaxation time is much less than the rotation period), assuming that the spin-spin and spin-lattice relaxation times are equal.

3. Given the above dissipation rates, we derived expressions for the diffusion coefficient (equations 72-74), which involve a constant of integration whose behavior in the low-temperature limit $(b \rightarrow \infty)$ is constrained.

4. We show that the boundary at $q=r_{2}$ is not a natural boundary for grains lacking dynamic symmetry. Thus, thermal flipping does occur in these grains.

5. We present results of long-time simulations of the internal grain dynamics using the Langevin equation for a few non-symmetric grain shapes. As expected, flipping does occur. For these simulations, we chose the constant of integration for the diffusion coefficient such that $q=r_{3}$ is a natural boundary. A first-principles analysis of Barnett relaxation is needed to definitively set the value of this constant. We also provide a practical definition of a flip.

Order-of-magnitude estimates of the spin-spin relaxation times are $T_{2} \sim 3 \times 10^{-11}$ to $3 \times 10^{-9} \mathrm{~s}$ for Barnett relaxation (Draine 1996) and $T_{2} \sim 3 \times 10^{-5}$ to $3 \times 10^{-4} \mathrm{~s}$ for nuclear relaxation (Lazarian \& Draine 1999b). Thus, our assumption of 
the low-frequency limit is much more severe for nuclear relaxation, limiting the applicability of our quantitative results to cases with low grain rotational speeds. We adopted the low-frequency limit in order to exploit analytical solutions to the modified Bloch equations (36)-(38). In future work, we will numerically integrate these equations to obtain results valid for all frequencies. We expect that the dissipation rate will be lower than the results derived here but that the main conclusion of this paper, namely the possibility of thermal flipping, will still hold.

We do not expect our assumption that $T_{1}=T_{2}$ to introduce significant error in the low-frequency limit, where saturation effects are negligible. Although $T_{1}$ and $T_{2}$ differ by several orders of magnitude for Barnett relaxation, the low-frequency limit breaks down only at relatively high rotational frequencies for which thermal flipping does not occur. In the Lazarian \& Draine (1999b) model of nuclear relaxation, the time-scale for spin-spin relaxation within the system of nuclear spins is of roughly the same magnitude as the time-scale for exchange between the nuclear and electron spin systems. The time-scale on which the electron spin system couples with the lattice is much shorter than the time-scale on which the nuclear and electron spin systems couple with each other, which in turn is much shorter than the time-scale on which the nuclear spin system couples directly with the lattice. Thus, the interaction between the nuclear spin system and the lattice is mediated by the electron spin system and the nuclear spin-lattice time-scale $T_{1}$ is of roughly the same magnitude as the nuclear spin-spin time-scale $T_{2}$.

In future work, we will extend our stochastic simulations to include the effects of systematic torques in order to quantify the impact of thermal trapping.

\section{ACKNOWLEDGEMENTS}

We are grateful to Bruce Draine and Alex Lazarian for helpful discussions.

\section{REFERENCES}

Draine B. T., 1996, in Roberge W. G., Whittet D. C. B., eds, Astronomical Society of the Pacific Conference Series Vol. 97, Polarimetry of the Interstellar Medium. p. 16 (arXiv:astro-ph/9603053)

Gardiner C. W., 2004, Handbook of Stochastic Methods, 3rd edn. Springer, Berlin

Gradshteyn I. S., Ryzhik I. M., 2015, Table of Integrals, Series, and Products, 8th edn. Academic Press, Amsterdam

Hall J. S., 1949, Science, 109, 166

Hall J. S., Mikesell A. H., 1949, AJ, 54, 187

Hiltner W. A., 1949a, Science, 109, 165

Hiltner W. A., 1949b, ApJ, 109, 471

Hoang T., Lazarian A., 2009, ApJ, 695, 1457

Lazarian A., Draine B. T., 1999a, ApJ, 516, L37

Lazarian A., Draine B. T., 1999b, ApJ, 520, L67

Lazarian A., Roberge W. G., 1997, ApJ, 484, 230

Press W. H., Teukolsky S. A., Vetterling W. T., Flannery B. P., 1992, Numerical Recipes in FORTRAN; The Art of Scientific Computing, 2nd edn. Cambridge University Press, New York, NY, USA

Purcell E. M., 1979, ApJ, 231, 404

Wangsness R. K., 1956, Physical Review, 104, 857

Weingartner J. C., 2009, ApJ, 690, 875

Weingartner J. C., Draine B. T., 2003, ApJ, 589, 289

This paper has been typeset from a $\mathrm{T}_{\mathrm{EX}} / \mathrm{IAT}_{\mathrm{E}} \mathrm{X}$ file prepared by the author. 